\title{
Barajar y dar de nuevo: reflexiones acerca de las prácticas de enseñanza en la pandemia
}

\author{
Shuffle and give again: reflections about teaching practices in \\ the pandemic
}

\author{
Rossana Viñas \\ https://orcid.org/0000-0001-6048-1537 \\ rovinas06@gmail.com \\ Centro de Investigación en Lectura y Escritura (CILE) | \\ Facultad de Periodismo y Comunicación Social | \\ Universidad Nacional de La Plata | Argentina
}

\section{RESUMEN}

El artículo es un ensayo que realiza un recorrido por distintas reflexiones desde la práctica y desde la teoría, sobre algunas prácticas docentes e institucionales llevadas adelante en la educación superior en el marco extraordinario devenido de la emergencia sanitaria por el Covid-19.

La pandemia nos inundó de incertidumbre, de preguntas y repreguntas acerca de cómo llevar adelante nuestras clases y nuestras prácticas institucionales en una virtualidad impuesta hasta la decisión y creatividad de poner en juego dispositivos y estrategias que rompieron estructuras y nos hicieron repensar usos y prácticas establecidas en la universidad pública. Una universidad pública como la argentina que nos interpela como trabajadorxs de ella, desde su historia y su compromiso, desde su valor educativo, político y social y que nos convierte en parte garantes de una educación superior para todxs en un país con proyecto igualitario y con real justicia social.

\section{ABSTRACT}

The article is an essay that makes a journey through different reflections from practice and from theory, on some teaching and institutional practices carried out in higher education in the extraordinary framework resulting from the health emergency caused by Covid-19.

The pandemic flooded us with uncertainty, with questions and cross-questions about how to carry out our classes and our institutional practices in a virtuality imposed up to the decision and creativity of putting into play devices and strategies that broke structures and made us rethink uses and practices established in the public university. A public university like Argentina's that challenges us as workers of it, from its history and its commitment, from its educational, political and social value and that makes us guarantors of a higher education for all in a country with an egalitarian project and with real social justice.

1 Doctora en Comunicación, Docente Investigadora de la Facultad de Periodismo y Comunicación Social, Universidad Nacional de La Plata (FPyCS-UNLP). Directora de Grado y Vicedirectora del Centro de Investigación en Lectura y Escritura (CILE) (FPyCS-UNLP).

PALABRAS CLAVE

pandemia, prácticas docentes, universidad, derecho a la educación superior.

\section{KEYWORDS}

pandemic, teaching practices, university, higher education right 


\title{
PRESENTACIÓN
}

\author{
La educación tiene como tarea movilizar todo lo necesario para que el \\ sujeto entre en el mundo y se sostenga en él, se apropie de los interrogantes que \\ han constituido la cultura humana, incorpore los saberes elaborados por los \\ hombres en respuesta a esos interrogantes... y los subvierta con respuestas pro- \\ pias, con la esperanza de que la historia tartajee un poco menos y rechace con \\ algo más de decisión, todo lo que perjudica al hombre. Esa es la finalidad de la \\ empresa educativa: que aquél que llega al mundo, sea acompañado al mundo. \\ Que sea introducido por quienes le han precedido... que sea introducido y no \\ moldeado, ayudado y no fabricado... (Meirieu, 1998, p. 70)
}

Comenzar este texto con el fragmento de Meirieu, de su texto Frankenstein Educador (1998), no es una decisión azarosa sino completamente deliberada que está relacionado con las experiencias como docente de la universidad pública, específicamente en la Facultad de Periodismo y Comunicación Social (FPyCS) de la Universidad Nacional de La Plata (UNLP), y mucho más en este contexto de emergencia sanitaria por el COVID-19 que seguimos transitando no sólo en la Argentina sino también en el mundo.

Estos tiempos de prácticas 24/7, de clases en pantuflas (Dussel, 2020), del ejercicio constante de la toma de decisiones, de reflexiones y repensar aquello que teníamos como establecido a rajatabla, nos ha invitado a ir y venir sobre nuestros pasos y nuestras prácticas pero con una mirada más amplia, para que profundicemos más allá de lo que está al simple alcance de la mano; desentrañando aquello que aparece en la superficie para problematizarlo desde su complejidad. (Achilli, 1990)

Ha sido y es un tiempo movilizante, retomando a Meirieu (1998), con incertidumbres, con preguntas y repreguntas que algunas fueron encontrando respuestas, aunque otras han quedado ahí, a la espera de continuar en la búsqueda. Porque el tránsito por éste ha servido para plantearnos nuevos interrogantes y tal vez, por qué no, parafraseando a Eldenstein (2021) y Meirieu (1998), a re-construirnos como 
sujetxs docentes, en un mundo social y educativo que pasó de la presencialidad a la virtualidad, y ahora, va camino a la hibridez o bimodalidad, conduciéndonos a una nueva normalidad educativa en la que el aula convivirá con las plataformas digitales.

\section{EDUCACIÓN TRANSMISIVA VS EDUCACIÓN EMANCIPADORA}

Comúnmente, hemos escuchado que la educación es un conjunto de contenidos determinados que como educadorxs debemos transmitir a lxs estudiantes para formarlxs; mucho más en la educación universitaria donde la transmisión ha estado tradicionalmente, ligada a la autoridad y la legitimidad: xn profesxr que transmite conocimiento en una clase magistral y construye su autoridad académica desde ese lugar (Eldenstein, 2021a, p. 3). Muchas veces, pensando que la pedagogía no importaba, porque lo esencial era el dominio del saber, de la disciplina para transmitirlo a xn otrx, independientemente de si esx otrx lo apropiara o no.

En la actualidad, esa idea ha ido transformándose tras debates que han comprendido contextualidades. La educación es más que eso: son procesos, son relaciones más complejas en las que la formación implica no "fabricar moldes", sino brindar herramientas para la relación con el mundo, con el campo profesional, para erigirse como sujetxs en ese mundo con otrxs. Movilizarlxs para que se incorporen al mundo, además, con un sentido crítico.

La educación es más que eso: son procesos, son relaciones más complejas en las que la formación implica no "fabricar moldes", sino brindar herramientas para la relación con el mundo, con el campo profesional, para erigirse como sujetxs en ese mundo con otrxs. Movilizarlxs para que se incorporen al mundo, además, con un sentido crítico.

En palabras de Meirieu (1998), Ixs docentes debemos formar personas (no objetos) para que adquieran destrezas para desenvolverse en la sociedad y para ello, debemos construir las condiciones reales para que otrxs aprendan (Meirieu, 1998), para que el proceso de enseñanza posibilite aprendizajes emancipatorios.

La emancipación, en un proceso de enseñanza dialógico, en la que la transmisión no es meramente reproductiva de lo sabido o conocido, sino que esa transmisión es interrumpida (Diker, 2004), es una conquista política y una lucha a favor de la comprensión comunicacional, del desmonte de discursos y el correspondiente desmantelamiento de narrativas dominantes. En el diálogo y en la interrupción, las pre- 
guntas y las re-preguntas llevan a propias re-significaciones y apropiaciones por parte $\mathrm{dxl}$ estudiante.

En ese sentido, justamente, debemos reflexionar y analizar nuestras prácticas docentes y nuestras prácticas de enseñanza, contextualizándolas histórica y socialmente (Eldenstein, 2000). Las pensamos, las planificamos, las diseñamos con objetivos y resonancias propias. Ahora bien, ¿pensamos en las de nuestrxs estudiantes? Incluso, sucede que pensamos y confiamos en que efectivamente, esas prácticas en nuestras clases, resonarán y no sucede. Entonces, ¿cómo hacemos sentido?, ¿cómo logramos la resonancia? Seguramente, en más de una oportunidad, en la contextualidad pandémica, estas preguntas han aparecido en nuestra cabeza, luego de mirar una y otra vez la pantalla del Zoom y sentir que tal vez, el desarrollo de la clase o la práctica pensada podría haber sido otro, y desafiándonos a la autocrítica y a la creatividad constante.

\section{DE LA SECUNDARIA A LA UNIVERSIDAD}

El pasaje de la escuela secundaria a la universidad es uno de los momentos más complejos en la vida de cualquier joven: tiene que elegir una carrera (muchas veces por propia decisión y otras, por mandato), adaptarse a nuevas reglas institucionales al ingresar a un mundo en cual debe tomar decisiones y manejarse de manera autónoma.

Asimismo, a una nueva alfabetización académica ya que ese ingreso demanda nuevas formas de leer y escribir: "los modos de leer y escribir -de buscar, adquirir, elaborar y comunicar conocimiento- no son iguales en todos los ámbitos". (Carlino, 2003, p.410)

Como docentes en la universidad, debemos tener en cuenta que xl joven que se halla en ese tránsito no sólo está cambiando de nivel educativo -con todo lo que ello implica-, sino que también está asistiendo a transformaciones personales, psicológicas y sociales que "requieren de una adaptación al nuevo sistema, tanto a nivel académico como social, para lograr la integración en la cultura universitaria". (Brito, 2012, p. 57)

En los estudios superiores, la participación en este nuevo espacio y en sus usos y prácticas suponen un estudiante autónomo y participativo; poseedor de un capital cultural, adquirido con anterioridad (Ezcurra, 2011, p. 54). Sin embargo, para esx joven ingresante, esta cultura le es ajena y la adaptación a ella es doble: académica e institucionalmente.

Si lo hace en los dos sentidos implicará el acceso total al mundo universitario, el haber aprendido y aprehendido el oficio de ser estudiante: el "éxito". En tanto, de producirse, xl estudiante enfrentará el denominado "fracaso".

Aunque si bien ha cambiado la idea de "estudiante ideal", en muchxs docentes aún persiste la idea de estudiante de tiempo completo al estudio, de background de conocimientos adquiridos en la cultura 
letrada y de competencias comunicativas pertinentes a las de un futurx profesional.

Aunque si bien ha cambiado la idea de "estudiante ideal", en muchxs docentes aún persiste la idea de estudiante de tiempo completo al estudio, de background de conocimientos adquiridos en la cultura letrada y de competencias comunicativas pertinentes a las de un futurx profesional.

Esxs docentes, entonces, trabajan en el aula partiendo de esa representación, sin prestar atención a que es necesaria la denominada "trasposición didáctica"; transformar el saber sabio en saber enseñado sin anteponer el ideal de estudiante. Todxs pueden aprender; todxs deben tener la oportunidad.

Sin embargo, y en clave de afirmar la educación superior como derecho y a la universidad desde una mirada inclusiva, la premisa sería partir del capital cultural real que posea -sea cual fuera éste- xl estudiante, y así lograr que éste pueda sortear las dificultades académicas que encuentre y de esta manera, adquiera el habitus organizativo y académico (Ezcurra, 2011, p. 55) que requiere la universidad.

$Y$ ahí reside la importancia del rol del docente recibiendo al recién llegado desde la hospitalidad y el acompañamiento de sus primeros pasos. Pensando en las particularidades, en las diferencias que cada unx presentan, entendiendo que cada unx trae consigo diferentes trayectorias educativas y sociales diversas que se ponen en juego dentro y fuera del aula. Comprendiendo, además que en el aula, hay diversidad y esa diversidad es la que la enriquece (Freire, 2006). Por lo que las clases de esa aula habitada por la diversidad, deben tenerlo en cuenta.

En relación a ello, en la actualidad de las aulas de la universidad pública, la heterogeneidad y la diversidad son su característica y al mismo tiempo, conforman parte de los debates educativos: ¿cómo hacemos para llegar y contener a todxs?

En relación a ello, en la actualidad de las aulas de la universidad pública, la heterogeneidad y la diversidad son su característica y al mismo tiempo, conforman parte de los debates educativos: ¿cómo hacemos para llegar y contener a todxs?

\section{LA DOCENCIA EN TIEMPOS DE COVID-19}

En tiempos de la emergencia sanitaria que vivimos desde marzo de 2020, la práctica docente y las prácticas de la enseñanza se han transformado radicalmente (Socolovsky, junio de 2020). Pasamos de una presencialidad absoluta, en un aula con bancos, pizarrón y escritorio 
(esa arquitectura conocida por todxs), con cuerpos interactuando a una virtualidad total desde nuestros hogares. Los distintos rincones de nuestras casas se convirtieron en el aula donde ejercimos nuestro rol docente de una manera completamente diferente.

de repente la casa (y a veces la habitación) son multifunción. (...) estamos dando clase en pantuflas (...) Lo que creo que hay que preguntarse es qué cambia en la clase cuando estamos en casa y los chicos en la suya, qué tipo de clase es esa, qué pasa con nuestras pedagogías, con nuestro trabajo docente cuando tenemos que lidiar cotidianamente con múltiples demandas domésticas y laborales, que ya eran un poco infinitas y se nos colaban por todas partes (preparar clases, corregir trabajos, reuniones extras) pero ahora se multiplican por mil. (Dusell, 2020)

Sobrecarga, desdibujamiento de tiempos y roles, organización, disponibilidad de TIC (Socolovsky, junio de 2020), entre tantos otros, pasaron a ser tema de debate, revisión y reflexión.

Tuvimos que construir el aula de la virtualidad. Tuvimos que ser creativxs, buscar la mejor manera de dar nuestros contenidos, en una construcción casuística. El aula que tal como lo mencionan Dusell y Caruso (1999), en La invención del aula, se conforma a partir de una estructura material y una comunicacional, tuvo que ser replanteada. No fue solo diseñar el aula, sino también ocuparla y habitarla para que nuestras prácticas de enseñanza hagan mella, desde otra estructura material y buscando los mejores canales y estrategias comunicativas.

Es claro que pasar por el aula y habitarla, siempre nos marca. Y eso fue lo que tuvimos que recrear en la virtualidad pero también tuvimos que aprender a salir del miedo a tener todo bajo control, a pensar otros modos, a darle nuestro toque personal en el andar mismo de ese aula (ahora Zoom, Meeting, etc.) y de la clase en sí misma (Dusell y Caruso, 1999).

\section{Es claro que pasar por el aula y habitarla, siempre nos marca. Y eso fue lo que tuvimos que recrear en la virtualidad pero tam- bién tuvimos que aprender a salir del miedo a tener todo bajo control, a pensar otros modos, a darle nuestro toque personal en el andar mismo de ese aula (ahora Zoom, Meeting, etc.) y de la clase en sí misma (Dusell y Caruso, 1999).}

Tomando ejemplos de prácticas docentes enuncio dos en los que me desempeño como docente: el Taller de Lectura y Escritura I en la Licenciatura en Comunicación Social y Prof. Titular en Estudios de Política y Sociedad I de la Tecnicatura Superior Universitaria en Comunicación Pública y Política; y uno, como gestión: la Dirección de Grado de la Secretaría Académica de la Facultad de Periodismo y Comunicación Social (UNLP). 
En ambos espacios, trabajamos desde una matriz político-ideológica inclusiva, pensando y sintiendo la educación superior como derecho humano y social.

En los espacios curriculares citados, nos ocupamos en profundidad de la lectura, la escritura y el análisis del texto-contexto-autxr, para comprender quién dijo qué, cómo, dónde y en qué momento. Asimismo, nos relacionamos con estudiantes de 1 er año y hacemos muchísimo hincapié -más allá de los contenidos de cada una- en la lectura y la escritura como herramientas y como prácticas necesarias para el campo profesional, siempre acompañando, docentes y adscriptxs, las trayectorias diversas de nuestrxs estudiantes (mucho más en este contexto especial), pero también haciendo ese importante anclaje en la educación superior como derecho humano y social.

La modificación de la Ley de Educación Superior en 2015 promulgó el ingreso irrestricto y el Estado se convirtió en garante de ello y de su gratuidad: Por otra parte, la Conferencia Regional de Educación Superior (CRES) 2018 declaró a la educación superior como "bien público y social, un derecho humano fundamental y una responsabilidad de los Estados" (Abratte, 2019, p. 71).

La modificación de la Ley de Educación Superior en 2015 promulgó el ingreso irrestricto y el Estado se convirtió en garante de ello y de su gratuidad: Por otra parte, la Conferencia Regional de Educación Superior (CRES) 2018 declaró a la educación superior como "bien público y social, un derecho humano fundamental y una responsabilidad de los Estados" (Abratte, 2019, p. 71).

En este contexto entonces, esa diversidad y la heterogeneidad que se mencionó anteriormente, son un desafío y una obligación ético-política como docentes. En este punto, aparece la conceptualización de una enseñanza que incluye en su seno la idea de hospitalidad y de acompañamiento de una otredad diversa y masiva, y teniendo en cuenta la alteridad; esa posibilidad de rupturas y de diálogos constantes (Eldenstein, 2021a, p. 3) para poder entonces, posibilitar que cada unx encuentre $y / o$ construya su propio camino.

En las materias que mencioné, y con estudiantes que nunca transitaron los pasillos de la Facultad, apelamos, por ejemplo, a videos y fotos del aula real en la que dábamos clases en la presencialidad (la 21 de la FPyCS) o a tomarnos diez o quinces minutos para charlar con ellxs e introducirnos a la clase. O en el caso de la Dirección de Grado, pensamos los coloquios virtuales de Trabajos Integradores Finales (TIF) con la magia de la compañía de la familia y amigxs, para celebrar juntxs ese momento tan especial en la vida de Ixs jóvenes y lxs no tan jóvenes. Asimismo, en el caso de la finalización de carrera, posibilitó que estudiantes que habían abandonado la idea de recibirse, con la eventualidad de la virtualidad, volvieran a nuestros pasillos digitales, 
a terminar sus estudios.

Retomando a Brailovsky (2020), la pregunta en aquel momento inicial de la pandemia, fue qué significaba mudar el aula presencial a la virtualidad, qué se perdía, qué se resignificaba; cómo poníamos el cuerpo allí... cómo creábamos corporeidad en ese "cuadrito" de la video-conferencia.

Entonces y tal como lo plantea este pedagogo, Ixs docentes nos erigimos en un doble sentido: como anfitrionxs y como arquitectxs.

\section{Entonces y tal como lo plantea este pedagogo, Ixs docentes nos erigimos en un doble sentido: como anfitrionxs y como arquitectxs.}

Arquitectxs porque planificamos, diseñamos, para elegir las mejores herramientas porque nos somos improvisadxs. $Y$ anfitrionxs para ser acogedorxs, para entender al otrx desde su realidad, porque no somos indiferentes, porque nos importan las relaciones (Brailovsky, 2020). Y en un momento de tanta incertidumbre, fuimos más allá de nuestras planificaciones que dialogaron con nuestrxs estudiantes pero también con la realidad pandémica.

Fuimos arquitectxs y anfitrionxs de una corporeidad posible en ese cuadrito de la video-conferencia, dándole la bienvenida a cada estudiante a nuestra Facultad o celebrando su título, en el último instante antes de ser graduadx para toda la vida de la UNLP.

Es así que Ixs docentes construimos didáctica y metodológicamente, no desde lo absoluto, sino desde lo casuístico:

el docente como sujeto que asume la tarea de elaborar una propuesta de enseñanza en la cual la construcción metodológica deviene fruto de un acto singularmente creativo de articulación entre la lógica disciplinar, las posibilidades de apropiación de ésta por parte de los sujetos y las situaciones y los contextos particulares que constituyen los ámbitos donde ambas lógicas se entrecruzan. (Edelstein, 1996, p. 85)

Porque no hay una receta didáctica absoluta y es por eso que debemos ampliar la mirada. Tal como menciona Edelstein (2021b), para analizar los sentidos en juego, de las acciones en una clase diseñada debemos pensar en las decisiones (siempre lo que un docente plantea en una clase tiene que ver con decisiones, conscientes o no); y en ellas: las macro-decisiones (las que se pueden anticipar cuando diseñamos la clase -intencionalidades, propósitos y adscripciones teórico-metodológicas-; y las micro-decisiones, que se van resolviendo en la clase (preguntas que nos van a formular, si Ixs estudiantes participan o no, entre otras).

Según Litwin (1997), la configuración didáctica es la manera que despliega xn docente para favorecer procesos de construcción de conocimiento en una clase. Los modos en que facilita los procesos de 
comprensión y apropiación de Ixs estudiantes al operar como mediadores entre Ixs estudiantes y el conocimiento.

$Y$ así fuimos transitando, sobre la prueba y el error, pero persistiendo, sin pretensiones del control sobre todo. En la virtualidad, como en el aula presencial, Ixs estudiantes hablan: desde sus silencios, desde su intervención en el chat, si muestran su rostro real -y su casa- o una foto o imagen, si prenden o no la cámara.

Daniel Berisso (2015, pp. 18-19) se refiere a ese silencio, que puede ser pensante, de nuestrxs estudiantes, no significa que no esté activo. Por lo que no debemos esperar algo a cambio, sino ayudar al otrx para que encuentre su propio camino.

¿Cuántas veces en el transcurrir de nuestras clases virtuales o al finalizar las mismas, hemos pensado si realmente nos han escuchado, si les hemos llegado... y de repente, aparecía un mail de algxn estudiante con la cámara apagada, aportando algún dato, un texto, algo que lo movilizó de nuestras palabras?

"El valor central de la labor analítica es el de volver a pensar la clase". (Eldenstein, 2021b, p. 9) Volver a mirarla críticamente desponjándose de ideas universalizantes, sin evaluaciones de los propios análisis desnaturalizando lo naturalizado y descotidianizando lo cotidiano. “El valor central de la labor analítica es el de volver a pensar
la clase". (Eldenstein, 2021 b, p. 9) Volver a mirarla críticamente
desponjándose de ideas universalizantes, sin evaluaciones de
los propios análisis desnaturalizando lo naturalizado y desco-
tidianizando lo cotidiano.

\section{PARA SEGUIR PENSANDO}

Tanto docentes como estudiantes hemos sido protagonistas y productorxs de contenidos en la virtualidad. Nos replanteamos lo macro y el microespacio de las clases, visibilizamos las distintas variables que condicionan que el acto de enseñanza y el acto de aprendizaje se lleven a cabo, las distintas herramientas que como educadorxs tenemos, entre tantas otras. Este tiempo extraordinario que vivimos, nos "movió el piso", vino a romper estructuras y nos permitió y nos invitó al mismo tiempo, a reflexionar sobre nuestras prácticas.

La universidad pública argentina actual es producto de los hitos (la Reforma del 18, la Ley de Gratuidad del 49, la modificación de la LES en 2015, entre otros) que la conformaron como una institución educativa con conciencia y compromiso por su función política, social y transformadora.

La enseñanza es un acto político, y desde la universidad pública argentina -con su tradición e historia-, en este contexto, Ixs docentes, "al estar presentes en el hogar de ese/a estudiante éramos/somos el Estado en su hábitat garantizando el derecho a la educación superior" (Viñas, Vena y Zangara, 2020, p. 5) a todxs; en el ingreso, la permanencia y el egreso. Y estuvimos, estamos y estaremos. 
La enseñanza es un acto político, y desde la universidad pública argentina -con su tradición e historia-, en este contexto, Ixs docentes, "al estar presentes en el hogar de ese/a estudiante éramos/somos el Estado en su hábitat garantizando el derecho a la educación superior" (Viñas, Vena y Zangara, 2020, p. 5) a todxs; en el ingreso, la permanencia y el egreso. Y estuvimos, estamos y estaremos.

El aula y la clase no son cajas herméticas; la realidad social, institucional, personal (tanto la de Ixs estudiantes como la de Ixs docentes) se filtra constantemente y ahí, aflora lo humano, el diálogo y el reconocimiento $\mathrm{dxl}$ otrx.

El reto hoy es cómo pensar la universidad de la pospandemia y con ella, todas y cada una de las prácticas que en la futura hibridez -que iremos construyendo por supuesto ese derecho que mencionamos sea siempre garantizado para todxs. Porque en que esxs todxs accedan, ingresen, permanezcan y egresen reside la verdadera justicia social. Y que la universidad que queremos, sea parte inseparable del país que anhelamos. 


\section{BIBLIOGRAFÍA}

Abratte, J.P. (2019). "Educación Superior y Derechos Humanos: reflexiones, apuestas y desafíos". En Derechos humanos y educación superior. Paraná, Entre Ríos, Argentina: Editorial Uader.

Achilli, E. L. (1990). “Antropología e investigación educacional. Aproximación a un enfoque constructivista indiciario". Rosario, Santa Fe, Argentina: Centro Rosario de Investigaciones en Ciencias Sociales, Fac. de Humanidades y Artes, UNR.

Berisso, D. (2015). ¿Qué clase de dar es el dar clase"? Alteridad, donación y contextualidad. Buenos Aires, Argentina: Antropofagia.

Brailovsky, D. (2020). "Las viejas tecnologías en tiempos de cuarentena”. Recuperado de https://www.youtube.com/watch?v=nhtdQjfc9YI

Brito, A. (dir.) (2010). Lectura, escritura y educación. Rosario, Santa Fe, Argentina: Flacso Argentina-Homo Sapiens Ediciones, Colección Pensar la Educación

Carlino, P. (2003). “Leer textos científicos y académicos en la educación superior: Obstáculos y bienvenida a una cultura nueva". En Uni-pluri/versidad, vol. 3, núm. 2, pp. 17-23. Recuperado de https://www.aacademica.org/paula.carlino/184

Diker, G. (2004). "Y el debate continúa. ¿Por qué hablar de transmisión?". En Frigerio, G. y Diker, G. (Comps.). La transmisión en las sociedades, las instituciones y los sujetos. Un concepto de la educación en acción. Buenos Aires, Argentina: Novedades educativas.

Dussell, I. (2020). "La clase en pantuflas". Recuperado de https://red.infd.edu. ar/wp-content/uploads/2020/05/VEP-audiovisual-Ficha-4-La-clase-en-pantuflas-Ines-Dussel.pdf

DUSSEL, I. y CARUSO, M. (1999). La invención del aula. Una genealogía de las formas de enseñar. Buenos Aires, Argentina: Santillana. Recuperado de http:// pedagogiaunrpsi.blogspot.com/2018/03/texto-la-invencion-del-aula-inesdussel.html

Edelstein, G. (1996). “Un capítulo pendiente: El método en el debate didáctico contemporáneo". En Camilloni y otras (1996). Corrientes didácticas contemporáneas. Buenos Aires, Argentina: Paidos.

(2021). Clase sincrónica en el marco de la materia Análisis de las Prácticas de Enseñanza de la Especialización en Docencia Universitaria de la Universidad Nacional de La Plata. 
(2021a, 2021b). Clases asincrónicas 1 y 4, en el marco de la materia Análisis de las Prácticas de Enseñanza de la Especialización en Docencia Universitaria de la Universidad Nacional de La Plata.

Ezcurra, A. M. (2011). Igualdad en la educación superior. Un desafío mundial. Los Polvorines, General Sarmiento, Buenos Aires, Argentina: Instituto de Estudios y Capacitación, Federación Nacional de Docentes Universitarios, Colección Educación, Serie Universidad.

Freire, P. (2006). Pedagogía de la autonomía. Saberes necesarios para la práctica educativa. México: Siglo XXI.

Litwin, E. (1997). Las configuraciones didácticas. Buenos Aires, Argentina: Paidós.

Meirieu, P. (1998). Frankenstein Educador. Barcelona, España: Laertes S. A. de Ediciones.

Socolovsky, Y. (junio de 2020). Panel sobre Docencias, desarrollado en el marco de las de las $3^{\circ}$ Jornadas sobre las Prácticas Docentes en la Universidad Pública UNLP. Recuperado de https://www.youtube.com/watch?v=97RXilMw0i8\&t=4057s

Viñas, R., Vena, L. Y Zangara, M. (2020). “Una metodología de enseñanza de la comunicación política durante la emergencia sanitaria por el COVID 19. Entre la teoría y la práctica profesional". En Actas de Periodismo y Comunicación, 6(2): IV COMCIS. Recuperado de https://perio.unlp.edu.ar/ojs/index.php/actas/article/view/6812

\section{BIBLIOGRAFÍA DE CONSULTA}

Edelstein, G. (2021a, 2021b). Clases asincrónicas en el marco de la materia Análisis de las Prácticas de Enseñanza de la Especialización en Docencia Universitaria de la Universidad Nacional de La Plata.

Terigi, F. (2004). “La enseñanza como problema político”. En Frigerio, G. y Diker, G. (Comps.). La transmisión en las sociedades, las instituciones y los sujetos. Un concepto de la educación en acción. Buenos Aires, Argentina: Novedades educativas. 